\title{
Purple glove syndrome caused by intravenous phenytoin: two case reports
}

CY Tsang ${ }^{1 *}$, MB, ChB, MRCSEd, Joanne YW Ng ${ }^{1}$, MB, BS, SH Yuen ${ }^{1}$, FRCSEd, FHKAM (Orthopaedic Surgery), IT Lau², FRCP, FHKAM (Medicine), YF Leung ${ }^{1}$, FRCSEd, FHKAM (Orthopaedic Surgery)

${ }^{1}$ Department of Orthopaedics and Traumatology, Tseung Kwan O Hospital, Hong Kong

2 Department of Medicine, Tseung Kwan O Hospital, Hong Kong

Hong Kong Med J 2019;25:487-9

*Corresponding author: tcy634@ha.org.hk

https://doi.org/10.12809/hkmj176974

\section{Case 1}

An 81-year-old woman was admitted in June 2014 to Tseung Kwan O Hospital with aspiration pneumonia. She had temporal lobe necrosis and absence seizure after radiotherapy for nasopharyngeal carcinoma. She was prescribed lifelong oral phenytoin with no adverse reaction reported to date. Oral phenytoin was changed to intravenous, $100 \mathrm{mg}$ every 8 hours, via a $22 \mathrm{G}$ catheter in her right wrist due to oropharyngeal dysphagia. The catheter had been changed several times due to blockage with no apparent extravasation. Erythematous swelling of the skin around previous cannula sites was treated as cellulitis by intravenous ciprofloxacin.

The following day the patient's right hand deteriorated and showed marked circumferential purplish discolouration, swelling, and gangrenous changes to the skin (Fig 1). Similar minor changes over the left hand were noted. She was afebrile and not septic. White cell count $\left(12.8 \times 10^{9} / \mathrm{L}\right)$ was mildly elevated and C-reactive protein was elevated at $196 \mathrm{mg} / \mathrm{L}$. Nonetheless, these results could be interpreted as being due to concurrent aspiration pneumonia.

Computed tomographic angiography revealed fluid collection in the patient's right hand up to the right forearm with reasonable arterial supply. Intravenous penicillin $G$ and ciprofloxacin were started. Serial clinical assessments prior to surgery showed no further deterioration. Emergency incision, drainage, and carpal tunnel release were performed. Findings were skin and fat necrosis, venous thrombosis and a large amount of clear fluid over the fascial layers of the palm and fingers. There was no turbid or dishwater discharge. The transverse carpal retinaculum was thickened and tight. Mild necrosis of the right-hand small muscles was noted. Cultures of intra-operative tissue samples were negative for micro-organisms.

On day 15 of intravenous phenytoin injection, the patient was diagnosed with purple glove syndrome (PGS). Intravenous phenytoin was stopped immediately and changed to valproate. The condition of the patient's left-hand improved rapidly but that of her right hand continued to deteriorate

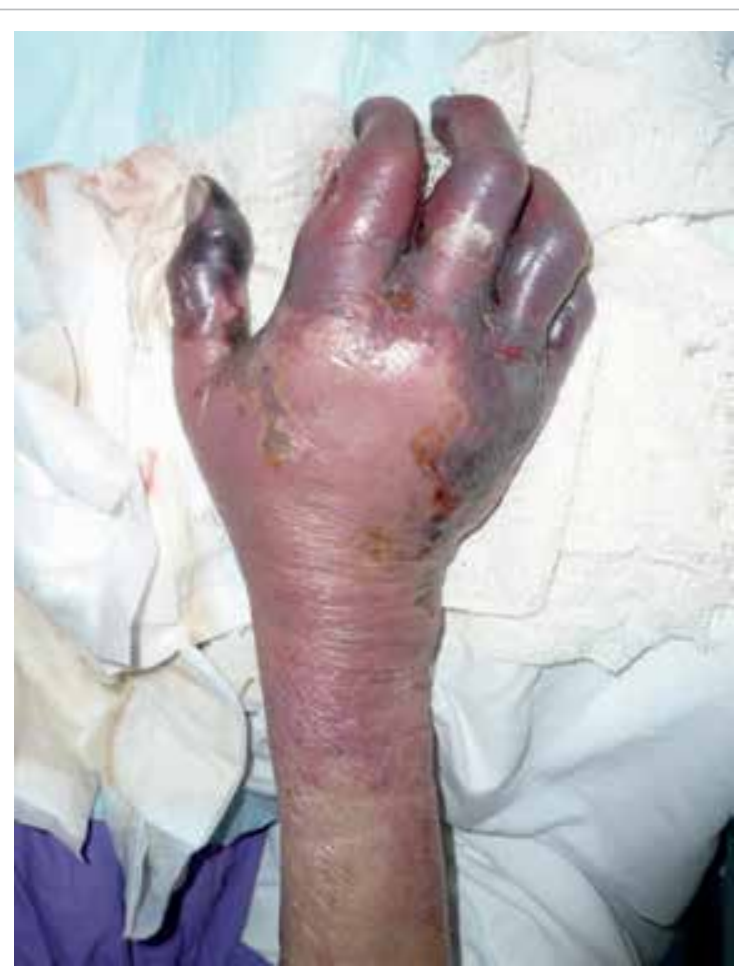

FIG I. Right hand of an 8I-year-old woman after 24 hours of intravenous phenytoin administration (100 mg every 8 hours)

(Fig 2) and necessitated below-elbow amputation. The amputation wound healed and she was referred for rehabilitation.

\section{Case 2}

An 87-year-old woman with brain metastasis from lung carcinoma was prescribed oral phenytoin for epilepsy. She was admitted to Tseung Kwan O Hospital in April 2016 with fever. She was prescribed $100 \mathrm{mg}$ phenytoin every 8 hours via a $22 \mathrm{G}$ catheter in her left hand due to potential dysphagia. She complained of painful swelling of her left hand after several doses of phenytoin. There was no extravasation of drug. The patient was afebrile and 

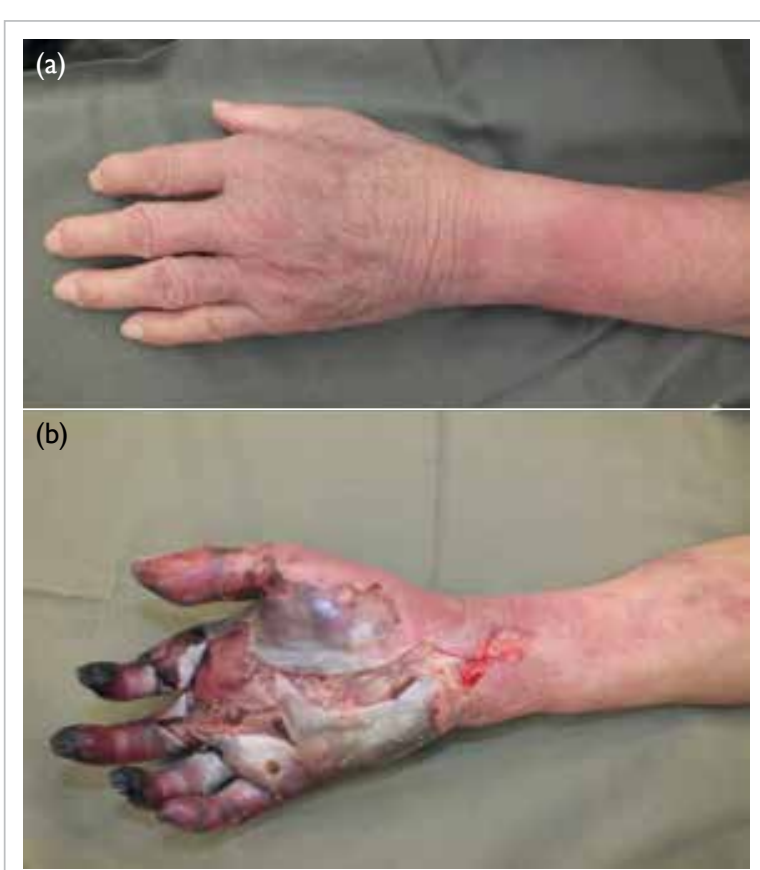

FIG 2. Left (a) and right (b) hands of an 8I-year-old woman after 15 days of intravenous phenytoin administration ( 100 mg every 8 hours)

not septic. Diffuse circumferential purplish, tender swelling of the distal left forearm and left hand was noted. The patient's hand was in intrinsic minus posture. There was no crepitation or fluctuance. The patient's left wrist and finger movements were limited by pain. Passive stretching of the fingers exacerbated her pain. Radial pulse was palpable with good capillary refill. White cell count $\left(12.4 \times 10^{9} / \mathrm{L}\right)$ and C-reactive protein were slightly elevated (17.2 $\mathrm{mg} / \mathrm{L}$ ).

The patient was suspected to have PGS of her left hand complicated by compartment syndrome. Intravenous phenytoin was stopped 1 day after injection and switched to valproate. Serial clinical assessments prior to surgery revealed no further deterioration. Emergency fasciotomy and carpal tunnel release were performed. Intra-operative findings were consistent with compartment syndrome and PGS. There was no evidence of necrotising fasciitis. Cultures of intra-operative tissue samples were negative for micro-organisms. Debridement and primary wound closure were performed 12 days after fasciotomy and the patient was transferred for rehabilitation.

Subsequently, the patient developed a breakthrough seizure and was prescribed intravenous phenytoin via her right hand because the physician did not notice that the patient had previously developed PGS as a complication of this treatment. She developed PGS of her right hand complicated by compartment syndrome. Emergency fasciotomy and carpal tunnel release of the right forearm and right hand were performed. Debridement and primary closure of wounds were performed 16 days after fasciotomy. In order to avoid future complications, a note was made in her medical records that she had an adverse reaction to phenytoin.

\section{Discussion}

Purple glove syndrome is an adverse reaction to intravenous phenytoin. Its incidence has been reported to range from $1.5 \%$ to $5.9 \% .^{1}$ Its manifestation includes pain, skin discolouration, blister formation, sloughing, ulceration, necrosis, and compartment syndrome at the injection site within hours of drug administration. Differential diagnoses include extravasation of the medication, cellulitis, peripheral vascular disease, venous thrombosis, and collagen vascular disease. ${ }^{2}$

The pathogenesis of PGS is poorly understood. It has previously been considered to be a result of extravasation of highly alkaline phenytoin $(\mathrm{pH}$ 12). ${ }^{3,4}$ However, PGS can occur in the apparent absence of extravasation. Histological examination has suggested thrombosis might play a role in pathogenesis. $^{3}$ To further complicate the matter, PGS has also been reported to be possible following oral phenytoin administration. ${ }^{4}$ There is no literature to date to correlate any intravenous complication with previous use of oral medication.

Risk factors for PGS after intravenous phenytoin administration include older age, female sex, peripheral vascular disease, sepsis, history of chronic debilitating disease, number of doses, higher dosage ( $>15-20 \mathrm{mg} / \mathrm{kg}$ body weight), higher infusion rate $(>25 \mathrm{mg} / \mathrm{min})$, and use of an intravenous catheter $<20 \mathrm{G} .{ }^{5}$

Initial management includes analgesia, elevation of the affected limb, compression, massage, and gentle heat. Phenytoin should be stopped and changed to an alternative antiepileptic. Most cases of PGS are mild and can be resolved with no long-term sequelae. Management is mainly supportive. However, PGS may progress to limb ischaemia and compartment syndrome. Frequent monitoring of neurovascular status is required. Vascular study should be performed if perfusion is compromised. ${ }^{5}$ Anticoagulation, thrombectomy or thrombolysis should be considered when arterial thrombosis is identified. Emergency fasciotomy should be performed for compartment syndrome. An unsalvageable limb should be amputated. Amputation has been reported to be necessary in $10.1 \%$ of cases. ${ }^{5}$ The use of regional blockade and hyaluronidase remains experimental. ${ }^{5}$

Purple glove syndrome may be prevented by avoiding phenytoin if there is an alternative 
agent with similar efficacy, for example valproate. Otherwise, oral phenytoin is preferred. If intravenous phenytoin is used, it should be administered via a larger catheter into a larger vein, at the correct dosage and infusion rate, and with a post-infusion saline flush. ${ }^{5}$

\section{Author contributions}

All authors contributed to the concept of study, acquisition and analysis of data, drafting of the article, and critical revision for important intellectual content. All authors had full access to the data, contributed to the study, approved the final version for publication, and take responsibility for its accuracy and integrity.

\section{Conflicts of interest}

The authors have no conflicts of interest to disclose.

\section{Funding/support}

This case report received no specific grant from any funding agency in the public, commercial, or not-for-profit sectors.

\section{Ethics approval}

The patient was treated in accordance with the Declaration of Helsinki. The patient provided informed consent for all procedures.

\section{References}

1. Rajabally H, Nageshwaran S, Russell S. An atypical case of purple glove syndrome: an avoidable adverse event. BMJ Case Rep 2012;2012. pii: bcr0120125653.

2. Lalla R, Malhotra HS, Garg RK, Sahu R. Purple glove syndrome: a dreadful complication of intravenous phenytoin administration. BMJ Case Rep 2012;2012. pii: bcr2012006653.

3. Okogbaa JI, Onor IO, Arije OA, Harris MB, Lillis RA. Phenytoin-induced purple glove syndrome: a case report and review of the literature. Hosp Pharm 2015;50:391-5.

4. Bhattacharjee P, Glusac EJ. Early histopathologic changes in purple glove syndrome. J Cutan Pathol 2004;31:513-5.

5. Garbovsky LA, Drumheller BC, Perrone J. Purple glove syndrome after phenytoin or fosphenytoin administration: review of reported cases and recommendations for prevention. J Med Toxicol 2015;11:445-59. 